\title{
Clinical Profile and Risk factors in Neonatal Sepsis
}

\author{
Fareedul. $\mathrm{H}^{1}$, Shamshad. $\mathrm{K}^{2}$,Prakash. $\mathrm{S}^{3}$ \\ 1,2,3 Yenepoya Medical college, Yenepoya University,Deralakatte, Mangalore-575018,India
}

\begin{abstract}
Objective : The aim of the study was to find the correlation of clinical profile and risk factors of neonatal sepsis with culture proven cases. Materials and Method: The study was conducted on 50 neonates a time based prospective study. All consecutive neonates fulfilling the inclusion and exclusion criteria are subjected to sepsis screening. Results: Among risk factors PROM and fetal distress were statistically significant with a p value of 0.009 respectively. Among the clinical features $80 \%$ of the neonates with mottling and grunting were proven sepsis which was statistically significant with a $p$ value of 0.015 respectively.Conclution: Neonatal sepsis is a common disease of newborn with non-specific symptomatology causing difficulty in the diagnosis. Early and prompt detection and appropriate treatment of neonatal sepsis can significantly reduce the morbidity and mortality
\end{abstract}

Keywords: Early-onset;neonate;Risk factors; clinical signs and symptoms

\section{INTRODUCTION}

Neonatal sepsis is the most common causes for neonatal mortality and morbidity, due to the delivery and postnatal care given in unhealthy environment and low socioeconomic state leading to maternal infection and neonatal sepsis. Therefore it is essential that we diagnose early onset sepsis using clinical signs and symptoms and rapid diagnostic techniques. Early onset sepsis can manifest as asymptomatic bacteremia, generalized sepsis, pneumonia or meningitis. Clinical signs are apparent in the first few hours of life and can have non-specific initial presentations. ${ }^{9}$ Even if there is one or two clinical signs and symptoms or if there is any maternal or neonatal risk factors is present even though the neonate may be asymptomatic sepsis screening is usually performed. Thus early suspicion and diagnosis of neonatal sepsis will help in early treatment with appropriate antibiotics which would reduce the morbidity and mortality in neonates. Septicemia is more common among infants whose mother had prolonged rupture of membrane which increases risk of contamination of amniotic fluid by organism from birth canal before deliver ${ }^{8}$ The present study was carried out to identify risk factors and clinical profile of neonatal sepsis in the neonatal unit.

Aim of the study was to find the correlation of clinical profile and risk factors of neonatal sepsis with culture proven cases.

\section{METHODS AND MATERIALS}

The study was carried out in Neonatal intensive care unit of Yenepoya Medical College, Yenepoya University, Deralakatte, and Mangalore. It is a prospective hospital based clinical study, over a period of one year from January 2013 to December 2013. All consecutive neonates fulfilling the inclusion and exclusion criteria are subjected to sepsis screening like serum procalcitonin, CRP, total count, gastric aspirate, peripheral smear and blood culture before starting treatment with antibiotics. 50 Neonates with suspected sepsis within the study period.

\section{Inclusion criteria}

All neonates with risk factors and clinical features of sepsis

Major risk factors

- $\quad$ PROM $>18 \mathrm{hrs}$

- Maternal fever $>38^{\circ} \mathrm{c}$ within 15 days

- Foul smelling liquor

- Fetal distress

Minor risk factors

- Low birth weight $<1500 \mathrm{gms}$

- Prematurity < 34 wks

- $\quad$ Birth asphyxia (APGAR <5)

- Maternal WBC > 15000

- Vaginal swab positive for GBS 


\section{Clinical signs and symptoms}

- Sclerema

- Lethargy

- Apnea

- Hypotonia

- Poor cry

- Breathlessness

- Irritability

- Grunting

- Poor feeding

- Vomiting

- Loose stools

- Temperature instability

- Mottling

\section{Exclusion criteria}

- Newborn babies with gestational age $<28$ wks

- Neonates with birth weight $<1000 \mathrm{gms}$

- Neonates with obvious malformation/congenital anomalies

- Outside born babies

Written and valid informed consent was taken from the parent of the subject included in the study and the disease process and importance of treatment was explained to them. The study design and proforma was approved by the institutional ethical committee. The patient declining to give consent were excluded in the study

A study proforma was designed and accordingly the study subject underwent detailed history, clinical examination and laboratory investigations. Maternal history was elicited and risk factors were noted in the proforma. Birth details were recorded as per babies' case sheet details. Birth weight was recorded using electronic weighing scale at birth. Clinical signs and symptoms were observed and documented by the treating doctor

Gestational assessment was done using modified Ballard's assessment scale. At the admission baby's vital signs were recorded followed by systemic clinical examination was done and findings were recorded in the proforma.

The data obtained from the study is entered in the master chart. Data was analyzed according to the statistical methods. Chi-square test has been used to study the significance of study parameters on categorical scale between groups.

\section{ANALYSIS AND INTERPRETATION OF DATA"}

The data was analyzed and interpreted by employing descriptive statistics. The statistical software namely SPSS 20.0, Stata 8.0, Med Calc 9.0.1 were used for the analysis of the data and Microsoft word and excel have been used to generate graph , table etc. Level of significance for the present study was taken as $\mathrm{P} \leq 0.05$.

Table 1: Distribution based on diagnosis

\begin{tabular}{|l|l|l|}
\hline DIAGNOSIS & FREQUENCY & PERCENT \\
\hline PROVEN SEPSIS & 16 & $32 \%$ \\
\hline PROBABLE SEPSIS & 34 & $68 \%$ \\
\hline
\end{tabular}

Table 2: Distribution of variables in relation to proven sepsis

\begin{tabular}{|c|c|c|c|c|}
\hline VARIABLES & & N=50 & PROVEN SEPSIS & P VALUE \\
\hline \multirow{2}{*}{ SEX } & MALE & $26(52 \%)$ & $7(26.5 \%$ & 0.200 \\
& FEMALE & $24(48 \%)$ & $9(37.5 \%)$ & \\
\cline { 2 - 4 } & PRETERM & $20(40 \%)$ & $9(45 \%)$ & 0.108 \\
\cline { 2 - 5 } & TERM & $30(60 \%)$ & $7(23 \%)$ & 0.204 \\
\hline \multirow{2}{*}{ TYPE OF DELIVERY } & LSCS & $16(32 \%)$ & $4(25 \%)$ & \\
\cline { 2 - 5 } & NVD & $34(685)$ & $12(35 \%)$ & 0.157 \\
\hline
\end{tabular}


Among the 50 neonates there was no statistical significance in comparison between sex, gestational age, type of delivery and birth weight in proven sepsis.

Table 3:Distributions of risk factors in relation to proven sepsis

\begin{tabular}{|l|l|l|l|}
\hline RISK FACTORS & N-50 & PEPSIS & P VALIE \\
\hline PROM & $14(28 \%)$ & $7(50 \%)$ & $\mathbf{0 . 0 0 9}(\mathbf{h s )}$ \\
\hline MATERNAL FEVER & $9(18 \%)$ & $5(55 \%)$ & 0.094 \\
\hline FOUL SMELLING LIQUOR & $12(24 \%)$ & $5(41 \%)$ & 0.410 \\
\hline FETAL DISTRESS & $24(48 \%)$ & $12(50 \%)$ & $\mathbf{0 . 0 0 9}(\mathrm{hs)}$ \\
\hline LOW BIRTH WEIGHT & $22(44 \%)$ & $10(45 \%)$ & 0.071 \\
\hline PRE-MATURITY & $20(40 \%)$ & $9(37 \% 0$ & .108 \\
\hline BIRTH ASPHYXIA & $5(10 \%)$ & $3(60 \%)$ & .157 \\
\hline MATERNAL WBC >15000 & $16(32 \%)$ & $4(25 \%)$ & .467 \\
\hline MECONIUM STAINED LIQUOR & $20(44 \%)$ & $9(37 \%)$ & .108 \\
\hline
\end{tabular}

Among the risk factors in our study group PROM had a significant $\mathrm{p}$ value of 0.009 and fetal distress had a $\mathrm{p}$ value of 0.009 which were statistically significant.

Table 4: Distribution of clinical features in relation to proven sepsis

\begin{tabular}{|c|c|c|c|}
\hline CLINICAL FEATURES & N-50 & PROVEN SEPSIS & P VALUE \\
\hline SCLEREMA & $7(14 \%)$ & $2(28 \%)$ & 0.834 \\
\hline LETHARGY & $18(36 \%)$ & $4(22 \%)$ & 0.266 \\
\hline APNEA & $6(12 \%)$ & $3(50 \%)$ & 0.314 \\
\hline HYPOTONIA & $4(8 \%)$ & $1(25 \%)$ & 0.754 \\
\hline POOR CRY & $10(20 \%)$ & $3(30 \%)$ & 0.880 \\
\hline POOR FEEDING & $28(56 \%)$ & $8(28 \%)$ & 0.558 \\
\hline RESPIRATORY DISTRESS & $24(48 \%)$ & $5(20 \%)$ & 0.104 \\
\hline VRUNTING & $5(10 \%)$ & $4(80 \%)$ & 0.725 \\
\hline TEMPERATURE INSTABILITY & $11(22 \%)$ & $4(36 \%)$ & 0.880 \\
\hline MOTTLING & $10(20 \%)$ & $3(30 \%)$ & $\mathbf{0 . 0 1 5}(\mathbf{h s})$ \\
\hline
\end{tabular}

Among the Clinical features which were present in our study group grunting and mottling had a $\mathrm{p}$ value of 0.015 respectively which were statistically significant.

This hospital based prospective study has observed and confirmed some known facts.

\section{Based on Gestational age, birth weight and sex distribution of the study group $(\mathrm{n}=50)$}

In this study, the age distributions was $42.8 \%$ of 21 preterm infants and $24.1 \%$ of 29 term infants had proven sepsis. Anderson -Berry et ${ }^{3}$ al in their study in 2008 in Carolina USA observed that sepsis is more common in preterm neonates. The results of our study were almost comparable with Raghavan et $\mathrm{al}^{2}$ and Tallur et $\mathrm{al}^{1}$

The higher proportion of term neonates compared to the preterm neonates in our study probably reflects difference in the population characteristics and the occurrence of the predisposing factors among them. Preterm are more susceptible to infection due to inherent defensive mechanism.

In this study male neonates with proven sepsis was $26.5 \%$ of the 26 and females neonates were $37.5 \%$ out of 24 were proven sepsis. IN the present study $40 \%$ neonates were with birth weight less than $<2.5 \mathrm{kgs}$ And our study showed $44 \%$ of 22 neonates with low birth were diagnosed with proven sepsis compared to $21.4 \%$ of 28 with normal birth weight were proven sepsis.

Anderson et al $^{3}$ also showed increased risk of neonatal sepsis with decrease in birth weight. Results in our study were almost comparable with Tallur et al ${ }^{1}$

\section{Based on risk factors in the study $\operatorname{group}(\mathrm{n}=50)$}

In our study $55 \%$ of the $9(18 \%)$ with maternal fever, $50 \%$ of the $14(28 \%)$ with prom,50\% 0f $24(48 \%)$ neonates with fetal distress, $45 \%$ of $22(44 \%)$ neonates with low birth weight, $45 \%$ of $20(40 \%)$ preterm, $41 \%$ of the $12(24 \%)$ with foul smelling liquor,45\% of $20(40 \%)$ had meconium stained liquor had proven sepsis and they were in significant in number. $28 \%$ of the $7(10 \%)$ with birth asphyxia, $25 \%$ of the $16(32 \%)$ with maternal wbc $>15000$ were diagnosed with proven sepsis and were less in number comparatively. Among the risk factors 
Prom and fetal distress were statistically significant with a p value of 0.009 respectively. The observation in our study is very similar to the Tallur et al ${ }^{1}$

In our study Prom was 50\% which was higher compared to $26 \%$ in Kuruvilla et al ${ }^{4}$

Foul smelling liquor was $41 \%$ in which was more than that observed in various studies but had closer results when compared to Raghavan et $\mathrm{al}^{2}$

The variation in the occurrence of intrapartum risk factors probably reflects differences in the rates of occurrence of the predisposing risk factors in various other studies.

\section{Based on clinical features in the study group(n-50)}

In our study poor feeding was the most common complaint and was seen in 28 neonates and $28 \%$ of the 28 were diagnosed with proven sepsis. Respiratory distress was seen in 24 neonates and $20 \%$ of them were proven sepsis.

Other clinical features were lethargy was 18 , vomiting was 11 , poor cry 10,10 had temperature instability 6 had apnea respectively which were not statistically significant 5 had mottling, grunting was seen in 5 .

Among the clinical features $80 \%$ of the neonates with mottling and grunting were proven sepsis which were statistically significant with a p value of 0.015 respectively, $50 \%$ of neonates with apnea and $36 \%$ of neonates with vomiting were proven sepsis.

Observation in our study were very close to various other studies.

\section{CONCLUSION}

Among risk factors PROM and fetal distress were statistically significant with a $\mathrm{p}$ value of 0.009 respectively. Among the clinical features $80 \%$ of the neonates with mottling and grunting were proven sepsis which was statistically significant with a p value of 0.015 respectively. Blood culture is the gold standard for diagnosing neonatal sepsis but it requires 48-72 hours Neonatal sepsis is a common disease of newborn with non-specific symptomatology causing difficulty in the diagnosis. Early and prompt detection and appropriate treatment of neonatal sepsis can significantly reduce the morbidity and mortality.

\section{REFERENCES}

[1]. Tallur.S.S,Kasturi.A.V,Shobha.D,Krishna.B.V.S.Clinico-bacteriological study of neonatal septicaemia in Hubli.IndianJPediatr.200;67(3):169-174.

[2]. Raghavan .M,Mondal.G.P,Bhatt.V,Srinivasan.S.Perinatal risk factors in neonatal infections.IndianJPediatr.1992;59:335-440.

[3]. Daniel.T.R,Praveen.K,Sandra.B.Neonatal sepsis in emergency department.Clinical Pediatric Emergrncy medicine.2008;9:160-168.

[4]. Kurien.A.M,Swati.P,Mary.J,Atanu.K.K.Bacterial Profile of sepsis in a Neonatal unit in south India.IndianPediatr.1998;35:851-858.

[5]. Betty.A.F,Daniel.F.S,Alice.S.W.Bailey \& Scott s Diagnostic Microbiology 11th ed,Mosby Publications:Elsevier;2002.

[6]. Nawshad.U.A,Azad.C,Mahbul.H,Gary.L.Darmstadt.Clinical bacteriological profile of neonatal septicaemia in a tertiary level Pediatric Hospital in Bangladesh.Indian Pediatrics 2002;39:1034-1038.

[7]. Bettychacko, Inderpreet.S.Early onset of Neonatal sepsis.IndianJPediatr.2005;72:23-26.

[8]. Jeffery.S, Gerdes,Richard.P.Early diagnosis and treatment of neonatal sepsis.IndianJPediatr.1998;65:63-71. 